\title{
OPEN SCIENCE: VOM RANDTHEMA ZUM MAINSTREAM?
}

\section{von Daniel Spichtinger}

Als ich 2012 bei der europäischen Kommission in der Generaldirektion Forschung meinen Dienst als Policy Officer antrat, wurde ich in den ersten Monaten nach meinem Betätigungsfeld gefragt. Nachdem ich angegeben hatte, dass ich für Open Access zuständig sei, kam die Gegenfrage „Und was machen Sie sonst noch?"

Anders als noch vor 7 Jahren sind Open Access, Open Data und Open Science keine Randthemen mehr, sondern wichtige Bestandteile nationaler, europäischer und internationaler Forschungspolitik. Dieses sogenannte „Mainstreaming“ ist größtenteils positiv zu sehen, hat aber auch Schattenseiten, wie Prof. Tal Yarkoni in seinem Blog-Beitrag „I hate open science“1 darstellt. Er kritisiert, dass der Terminus „Open Science“ mittlerweile von vielen verschiedenen Stakeholdern mit unterschiedlichen Bedeutungen verwendet wird. So sehen sich z.B. sowohl der große Wissenschaftsverlag Elsevier $^{2}$ als auch dessen Kritikerlnnen, wie der Paläontologe Jon Tennant ${ }^{3}$, als UnterstützerInnen von Open Science.

Auch wenn es also unterschiedliche Interpretationen des Begriffes gibt, lässt sich feststellen, dass zumindest einige Bestandteile von Open Science, wie Open Access zu wissenschaftlichen Forschungspublikation oder Open Data, auf der Ebene der Politik mittlerweile in vielen EU-Staaten verankert sind, was sich auch in den Anforderungen von nationalen und internationalen Forschungsförderungseinrichtungen an Fördernehmerlnnen (in Österreich wie z.B. des FWF ${ }^{4}$ ) niederschlägt.

Während also Open Access und Open Data bzw. Datenmanagement immer mehr in die Drittmittelförderung integriert werden, ist die Implementierung an Universitäten im deutschsprachigen Raum noch recht uneinheitlich. So kommt eine Analyse der OA-Policies von 47 deutschen

DOI: https://doi.org/10.31263/voebm.v72i2.3156

(c) Daniel Spichtinger

Dieses Werk ist lizenziert unter einer

Creative-Commons-Lizenz Namensnennung 4.0 International 
Universitäten und 5 Fachhochschulen zu dem Ergebnis, dass sich wichtige Themen wie Embargoperioden, Monitoring oder die Integration von Open Access-Schulungen in Lehrpläne in keiner einzigen dieser Leitlinien wiederfinden. ${ }^{5}$ Auf die österreichische Situation bezogen lassen sich durchaus positive Aspekte hervorheben: so sind an der Universität Wien nach eigenen Angaben bereits jetzt 57\% aller wissenschaftlichen Artikel in Plan S-konformen Publikationsorganen veröffentlicht worden; ${ }^{6}$ zusätzlich gibt es seit 2018 an vier österreichischen Universitäten (Medizinische Universität Wien, Universität für Musik und darstellende Kunst Wien MDW, TU Wien und Universität Graz) dezidierte Forschungsdatenmanagement-Policies. ${ }^{7}$ Eine starke und einheitliche Verankerung von Open Science-Elementen an allen österreichischen Hochschulen wird es dennoch wohl nur dann geben, wenn diese nicht nur verpflichtender Teil der Leistungsvereinbarungen des BMBWF mit allen 22 öffentlichen Universitäten sind (inkl. entsprechender Sanktionen bei Nichterfüllung) sondern auch in die Evaluierung individueller Wissenschaftlerlnnen einfließen, z.B. durch eine konsequente Implementierung der San Francisco Erklärung zur Forschungsevaluation (DORA). ${ }^{8}$ Um Open Science in Beurteilungskriterien zu integrieren, kann zum Beispiel die von einer EU-ExpertInnengruppe entwickelte Open Science Career Assessment Matrix $(\mathrm{OS}-\mathrm{CAM})^{9}$ dienen. Dies muss natürlich durch die nötigen Unterstützungsmaßnahmen (z.b. Training und die Bereitstellung umfassender Informationsunterlagen) flankiert werden. ${ }^{10} \mathrm{Im}$ Idealfall sollten spezifische Open Science-Policies mit den betroffenen WissenschaftlerInnen und der Einbeziehung von Open Science-ExpertInnen co-kreiert werden.

Auf europäischer Ebene ist es geplant, Open Science als „modus operandi“ für das neue Forschungsrahmenprogramm Horizon Europe zu implementieren. Anzunehmen ist, dass die konkreten Verpflichtungen auf den Bestimmungen in Horizon 2020 aufbauen werden, welche ja bereits eine Verpflichtung zu Open Access sowie Open Data nach dem Prinzip „So offen wie möglich, so geschlossen wie nötig" beinhalten. ${ }^{11}$

Was die Ebene der Mitgliedsstaaten angeht, wäre es wünschenswert, bestehende Initiativen, welche bereits Teile von Open Science beinhalten - im Besonderen die Priorität 5 des europäischen Forschungsraums ${ }^{12}$, die Empfehlung der Kommission zu Zugang zu und Aufbewahrung von wissenschaftlicher Information (2012, revidiert 2018) sowie der Ratsbeschluss zum Thema Open Science aus dem Jahr 2016 in einer kohärenten Open Science-Strategie zu bündeln, welche die Form einer Richtlinie ${ }^{13}$ (anstatt unverbindlicher Empfehlungen) annehmen sollte. Teil davon sollte die Entwicklung und Implementierung nationaler Open Science Strategien 
mit der Definition klarer Meilensteine sein. Unabhängig davon, ob eine verbindlichere Rechtsakte auf europäischer Ebene zustande kommt, wäre Österreich auf jeden Fall gut beraten, eine solche nationale Open Science-Strategie zu entwickeln und dabei das Open Science Network Austria (OANA) ${ }^{14}$ einzubeziehen.

Die Entwicklung von Guidelines für nationale Open Science-Policies wird gemeinsam mit der Entwicklung von Anreizen für Forscherlnnen und der Weiterentwicklung von Metriken in einem Gedankenaustausch der EU-Mitgliedsländer zum Thema Open Science (eine sogenannte „Mutual Learning Excercise) als Priorität für die Fortentwicklung von Open Science genannt. Aus dieser „Übung zum gemeinsamen Lernen“ sei auch das Schlusswort für meinen Artikel entnommen: Open Science ist mehr als Open Access und Open Data; es ist eine Art, die Welt zu betrachten, mit der Absicht, eine bessere Gesellschaft aufzubauen. "15

Mag. Daniel Spichtinger, M. A. ORCID: https://orcid.org/0000-0002-9601-8592 Ludwig Boltzmann Gesellschaft, Open Innovation in Science Center E-Mail: daniel.spichtinger@lbg.ac.at

\section{Literatur}

Declaration on Research Assessment (DORA): https://sfdora.org/ Elsevier, Open Science: https://www.elsevier.com/about/open-science Enago Academy (2019). Elsevier's Open Access Controversy: German Researchers Resign to Register Protest (19. Juli). https://www.enago. com/academy/move-boycott-elsevier-journals-deal-near-dead/

European Commission, European research area (ERA): https://ec.europa. eu/info/research-and-innovation/strategy/era_en

European Commission (2017). Evaluation of Research Careers fully acknowledging Open Science Practices; Rewards, incentives and/or recognition for researchers practicing Open Science. European Union. https://doi.org/10.2777/75255

European Commission (2018). MLE on Open Science: Summary Article - Enabling Systemic Change through Mutual Learning. https://rio. jrc.ec.europa.eu/en/library/mle-open-science-summary-article-enabling-systemic-change-through-mutual-learning

Europäische Union, Verordnungen, Richtlinien und sonstige Rechtsakte: https://europa.eu/european-union/eu-law/legal-acts_de 
FWF Der Wissenschaftsfonds, Open Access Policy: https://www.fwf.ac.at/ en/research-funding/open-access-policy/

Andreas Hübner \& Christina Riesenweber (2018). Datentabelle Inhalte der Open-Access-Policies deutscher Hochschulen [Data set]. Zenodo. http://doi.org/10.5281/zenodo.1291855

Siehe Sabina Leonelli, Daniel Spichtinger \& Barbara Prainsack (2015). Sticks and carrots: encouraging open science at its source. Geo: Geography and Environment 2(1), 12-16. https://doi.org/10.1002/geo2.2 Open Science Network Austria (OANA): https://oana.at/

Rita Pinhasi, Brigitte Kromp \& Guido Blechl (2019). Are we plan-S ready? Level of Compliance at the University of Vienna. Zenodo. http://doi. org/10.5281/zenodo.3258038

Jon Tennant (2018). Elsevier are corrupting open science in Europe. Guardian (29. Juni). https://www.theguardian.com/science/political-science/2018/jun/29/elsevier-are-corrupting-open-science-in-europe oder

Tal Yarkoni (2019). I hate open science (13. Juli). http://www.talyarkoni. org/blog/2019/07/13/i-hate-open-science/

1 Tal Yarkoni (2019). I hate open science (13. Juli). http://www.talyarkoni.org/blog/2019/07/13/i-hate-open-science/ (28.09.2019)

2 Elsevier, Open Science: https://www.elsevier.com/about/open-science (28.09.2019)

3 Siehe Jon Tennant (2018). Elsevier are corrupting open science in Europe. Guardian (29. Juni). https://www.theguardian.com/science/political-science/2018/jun/29/elsevier-are-corrupting-open-science-in-europe oder Enago Academy (2019). Elsevier's Open Access Controversy: German Researchers Resign to Register Protest (19. Juli). https://www. enago.com/academy/move-boycott-elsevier-journals-deal-near-dead/

4 FWF Der Wissenschaftsfonds, Open Access Policy: https://www.fwf. ac.at/en/research-funding/open-access-policy/ (28.09.2019)

5 Andreas Hübner \& Christina Riesenweber (2018). Datentabelle Inhalte der Open-Access-Policies deutscher Hochschulen [Data set]. Zenodo. http://doi.org/10.5281/zenodo.1291855

6 Rita Pinhasi, Brigitte Kromp \& Guido Blechl (2019). Are we plan-S ready? Level of Compliance at the University of Vienna. Zenodo. http:// doi.org/10.5281/zenodo.3258038

7 Policy für Forschungsdatenmanagement der Medizinischen Universität Wien: https://www.meduniwien.ac.at/web/rechtliches/policy-fuerforschungsdatenmanagement/; Policy für Forschungsdatenmanagement an der TU Wien: https://www.tuwien.at/forschung/fti-support/ 
forschungsdaten/forschungsdatenmanagement/policy/; mdw - Richtlinie des Rektorats zum Forschungsdatenmanagement: https://www. mdw.ac.at/upload/MDWeb/forschungsfoerderung/downloads/FDM_ Policy_mdw_DE_20171128endR_MB-01.pdf; Forschungsdatenmanagement-Policy derUniversität Graz: https://static.uni-graz.at/fileadmin/strategische-entwicklung/Dateien/FDM-Policy_DE_FINAL_Layout.pdf (04.10.2019)

8 Declaration on Research Assessment (DORA): https://sfdora.org/

9 European Commission (2017). Evaluation of Research Careers fully acknowledging Open Science Practices; Rewards, incentives and/or recognition for researchers practicing Open Science. European Union. https://doi.org/10.2777/75255

10 Siehe Sabina Leonelli, Daniel Spichtinger \& Barbara Prainsack (2015). Sticks and carrots: encouraging open science at its source. Geo: Geography and Environment 2(1), 12-16. https://doi.org/10.1002/geo2.2

11 Artikel 29.2 und 29.3. Im Fördervertrag mit der Europäischen Kommission (Model Grant Agreement).

12 European Commission, European research area (ERA): https://ec.europa.eu/info/research-and-innovation/strategy/era_en

13 Eine Richtlinie ist ein Rechtsakt, in dem ein von allen EU-Ländern zu erreichendes Ziel festgelegt wird. Es ist jedoch Sache der einzelnen Länder, eigene Rechtsvorschriften zur Verwirklichung dieses Ziels zu erlassen. Siehe Europäische Union, Verordnungen, Richtlinien und sonstige Rechtsakte: https://europa.eu/european-union/eu-law/legal-acts_de

14 Open Science Network Austria (OANA): https://oana.at/

15 Bart Dumolyn, Policy Advisor on Open Science and Responsible Research and Innovation for the Flemish Government. Eigenübersetzung. Siehe European Commission (2018). MLE on Open Science: Summary Article - Enabling Systemic Change through Mutual Learning. https:// rio.jrc.ec.europa.eu/en/library/mle-open-science-summary-article-enabling-systemic-change-through-mutual-learning 